\title{
Management of Spodoptera litura in Soybean through Biorational Approaches
}

\author{
M.R. Misal ${ }^{1 *}$, B.V. Patil ${ }^{1}$ and P.B. Khaire ${ }^{2}$ \\ ${ }^{1}$ Department of Agriculture Entomology, ${ }^{2}$ Department of Plant Pathology, College of \\ Agriculture, Badnapur, VNMKV Parbhani-431202, India \\ *Corresponding author
}

A B S T R A C T

\section{Keywords \\ Biorational, S.litura, Chlorpyriphos 20EC \\ Trap crop (castor), Intercrop (sorghum) \\ Article Info \\ Accepted: \\ 04 November 2018 \\ Available Online: \\ 10 December 2018}

Bio-rationals studies were carried out for Spodoptera litura in soybean with eight treatments. The results found that significantly reduction in S.litura population was found in experimental plots treated with chlorpyriphos 20EC @2ml/lit compare to rest of treatments. Chlorpyriphos 20EC @2ml/lit was statistically superior in controlling S.litura population and at par with NSKE 5\% and Neem oil $5 \%$. Trap crop (castor) found effective in reduction of S.litura population. It was statistically superior compare to rest of the treatments and at par with Pongamia oil $2 \%$ and intercrop (sorghum).

\section{Introduction}

Soybean [Glycine max (L.)] Merrill is one of the most important leguminous crop belonging to family Leguminoceae, sub-family Papilionoceae. It is the world's largest source of animal protein feed and the second largest source of vegetable oil.

It is considered as pulse crop but due to high oil content, now it is placed in oilseeds category. It originated from China (Nagata, T. 1960) where it also called 'yellow jewel' and introduced in India during 1870-80 (Andole, 1984).
Soybean crop attracts about 380 species of insects in many parts of the world (Luckmann, 1971). Only about a dozen minor insect pests were recorded on soybean in India in the early seventies, while in 1997 this number has increased to an alarming figure of 270 , besides 4 other invertebrates and 10 vertebrates (Singh, 1999). In Maharashtra, particularly in Marathwada 19 species of insects have been identified attacking this crop (Munde, 1980). Among them leaf miner (Aproaerema modicella Devanter), stem fly (Melanagromyza sojae Zehnter), girdle beetle (Obereopsis brevis Swedenboard), leaf eating caterpillar (Spodoptera litura Fab.), 
Helicoverpa armigera (Hubner), green semilooper (Chrysodeixis acuta Walker) and sucking insect pests such as whitefly (Bemisia tabaci Gennadius), aphid (Aphis craccivora Koch) and Leaf hopper (Empoasca Spp.) are important. Yellow mosaic virus (YMV) transmitted by whitefly poses a serious limitation in soybean cultivation in the northern India (Adak et al., 2012).

The Indian neem tree, Azadirachta indica (Meliaceae), is a promising source of botanical insecticides. Due to their relative selectivity, neem products can be recommended for many integrated pest management programs (Biswas et al., 2002).

\section{Materials and Methods}

The present investigation will be carried out in field condition on the field of Department of Entomology, College of Agriculture, Badnapur during Kharif, 2017.

The experiment will be laid out in Randomized Block Design (RBD) in three replications with a plot size of $5.0 \times 3.0 \mathrm{~m}$. leaving a gang way of 1 meter around plots. The soybean variety MAUS-71 will be sown at a spacing of $45 \times 5 \mathrm{~cm}$. Normal sowing will perform during Kharif 2017 by following recommended package of practices. The treatments as detailed it will imposed with Knapsack sprayer (Hydraulic sprayer) using a spray fluid of 500 lit/ha. The first spray will given at 30 days after germination (DAG) when crop had adequate population of insect and second spraying will be given at 45 days after germination (DAG). Observation on larval population of leaf eating caterpillar was recorded at three randomly selected spots on one meter length crop in each treatment leaving the border rows. Larval count was made by shaking the plant gently over a white cloth placed between rows. The population data were transformed to $\sqrt{ } \mathrm{x}+0.5$ value before analysis. To compare the efficacy of treatment both standard check as well as untreated check will be maintain.

The need based application of Neem oil 5\% will be given as and when needed after second spraying.

Treatments detail, NSKE 5\%, Neem oil 5\%, Pongamia oil $2 \%$, mechanical control, trap crop (castor), intercrop (sorghum), chlorpyriphos 20EC @2ml/lit and untreated control.

\section{Results and Discussion}

\section{Spodoptera litura}

The initial larval population of S.litura before imposing the treatment ranged from 4.2 to 5.3 larvae per $\mathrm{mrl}$ and differences were nonsignificant. First day after $I^{\text {st }}$ spray significat reduction of S.litura population is observed. At fifth day after spray chlorpyriphos 20EC @ $2 \mathrm{ml} /$ lit recorded the least larval population (2.3 larvar/mrl) which was significantly superior over the control followed by NSKE 5\% (2.7 larvar/mrl) and Neem oil 5\% (2.8 larvae/mrl). However, all treatments were significantly superior over untreated control, but pongamia oil $2 \%$ treated plot recorded highest larval population of 3.2 larvae/mrl. At seventh day after spraying similar trend was noticed.

The lowest S.litura were recorded in mechanical control $1^{\text {st }}, 3^{\text {rd }}, 5^{\text {th, }}$ and $7^{\text {th }}$ day after $\mathrm{I}^{\mathrm{st}}$ mechanical controls. Trap crop (castor) were found most effective in reducing S.litura larvae as shown in (Table 1 and Fig. 1). At fourteenth days after spray the chlorpyriphos 20EC@2ml/lit recorded the lowest larval population (3.1larvae/mrl) compared to untreated control and was superior. Untreated control recorded the highest population of (4.8 larvae/mrl). 
Table.1 Management of S.litura on soybean through bio-rational approaches

\begin{tabular}{|c|c|c|c|c|c|c|c|c|c|c|c|c|c|c|}
\hline \multirow{3}{*}{$\begin{array}{l}\text { Tr. } \\
\text { No }\end{array}$} & \multirow[t]{3}{*}{ Treatments } & \multicolumn{13}{|c|}{ Average larvae/mrl } \\
\hline & & \multicolumn{7}{|c|}{ First spray } & \multicolumn{6}{|c|}{ Second spray } \\
\hline & & 1DBS & 1DAS & 3DAS & 5DAS & 7DAS & 10DAS & 14DAS 1DBS & 1DAS & 3DAS & 5DAS & 7DAS & 10DAS & 14DAS \\
\hline$\overline{T_{1}}$ & NSKE $5 \%$ & $\begin{array}{c}5.0 \\
(2.34)\end{array}$ & $\begin{array}{c}4.8 \\
(2.29)\end{array}$ & $\begin{array}{c}4.2 \\
(2.17)\end{array}$ & $\begin{array}{c}2.7 \\
(1.77)\end{array}$ & $\begin{array}{c}0.9 \\
(1.18)\end{array}$ & $\begin{array}{c}1.6 \\
(1.42)\end{array}$ & $\begin{array}{c}3.3 \\
(1.96)\end{array}$ & $\begin{array}{c}3.1 \\
(1.90)\end{array}$ & $\begin{array}{c}2.7 \\
(1.77)\end{array}$ & $\begin{array}{c}2.0 \\
(1.58)\end{array}$ & $\begin{array}{c}0.6 \\
(1.02)\end{array}$ & $\begin{array}{c}1.4 \\
(1.39)\end{array}$ & $\begin{array}{c}2.8 \\
(1.81)\end{array}$ \\
\hline $\mathbf{T}_{2}$ & Neem oil 5\% & $\begin{array}{c}5.2 \\
(2.39)\end{array}$ & $\begin{array}{c}5.0 \\
(2.34)\end{array}$ & $\begin{array}{c}4.2 \\
(2.15)\end{array}$ & $\begin{array}{c}2.8 \\
(1.81)\end{array}$ & $\begin{array}{c}0.9 \\
(1.18)\end{array}$ & $\begin{array}{c}1.6 \\
(1.43)\end{array}$ & $\begin{array}{c}3.6 \\
(2.01)\end{array}$ & $\begin{array}{c}3.3 \\
(1.96)\end{array}$ & $\begin{array}{c}2.8 \\
(1.81)\end{array}$ & $\begin{array}{c}2.1 \\
(1.61)\end{array}$ & $\begin{array}{c}0.8 \\
(1.13)\end{array}$ & $\begin{array}{c}1.6 \\
(1.43)\end{array}$ & $\begin{array}{c}3.0 \\
(1.87)\end{array}$ \\
\hline $\mathrm{T}_{3}$ & Pongamia oil $2 \%$ & $\begin{array}{c}5.3 \\
(2.41)\end{array}$ & $\begin{array}{c}5.1 \\
(2.36)\end{array}$ & $\begin{array}{c}4.2 \\
(2.15)\end{array}$ & $\begin{array}{c}3.2 \\
(1.92)\end{array}$ & $\begin{array}{c}1.1 \\
(1.27)\end{array}$ & $\begin{array}{c}1.9 \\
(1.54)\end{array}$ & $\begin{array}{c}3.9 \\
(2.09)\end{array}$ & $\begin{array}{c}3.6 \\
(2.01)\end{array}$ & $\begin{array}{c}3.0 \\
(1.87)\end{array}$ & $\begin{array}{c}2.3 \\
(1.68)\end{array}$ & $\begin{array}{c}0.8 \\
(1.13)\end{array}$ & $\begin{array}{c}1.9 \\
(1.54)\end{array}$ & $\begin{array}{c}3.3 \\
(1.95)\end{array}$ \\
\hline $\mathbf{T}_{4}$ & Mechanical control. & $\begin{array}{c}5.3 \\
(2.17)\end{array}$ & $\begin{array}{c}0.5 \\
(0.97)\end{array}$ & $\begin{array}{c}0.6 \\
(1.02)\end{array}$ & $\begin{array}{c}1.0 \\
(1.22)\end{array}$ & $\begin{array}{c}1.4 \\
(1.39)\end{array}$ & $\begin{array}{c}2.3 \\
(1.68)\end{array}$ & $\begin{array}{c}4.1 \\
(2.12)\end{array}$ & $\begin{array}{c}0.3 \\
(0.91)\end{array}$ & $\begin{array}{c}0.6 \\
(1.02)\end{array}$ & $\begin{array}{c}0.8 \\
(1.13)\end{array}$ & $\begin{array}{c}1.4 \\
(1.38)\end{array}$ & $\begin{array}{c}2.1 \\
(1.59)\end{array}$ & $\begin{array}{c}3.4 \\
(1.98)\end{array}$ \\
\hline $\mathbf{T}_{5}$ & Use of trap crop, castor. & $\begin{array}{c}4.2 \\
(2.17)\end{array}$ & $\begin{array}{c}4.2 \\
(2.17)\end{array}$ & $\begin{array}{c}4.1 \\
(2.13)\end{array}$ & $\begin{array}{c}3.7 \\
(2.02)\end{array}$ & $\begin{array}{c}3.0 \\
(1.87)\end{array}$ & $\begin{array}{c}2.7 \\
(1.74)\end{array}$ & $\begin{array}{c}2.1 \\
(1.61) /\end{array}$ & $\begin{array}{c}1.9 \\
(1.54)\end{array}$ & $\begin{array}{c}1.7 \\
(1.44)\end{array}$ & $\begin{array}{c}1.6 \\
(1.42)\end{array}$ & $\begin{array}{c}1.4 \\
(1.36)\end{array}$ & $\begin{array}{c}1.3 \\
(1.34)\end{array}$ & $\begin{array}{c}1.1 \\
(1.27)\end{array}$ \\
\hline $\mathrm{T}_{6}$ & $\begin{array}{l}\text { Intercrop (4 rows of soybean } \mathrm{x} \\
2 \text { rows of sorghum). }\end{array}$ & $\begin{array}{c}4.2 \\
(2.17)\end{array}$ & $\begin{array}{c}4.2 \\
(2.17)\end{array}$ & $\begin{array}{c}4.2 \\
(2.17)\end{array}$ & $\begin{array}{c}3.8 \\
(2.07)\end{array}$ & $\begin{array}{c}3.1 \\
(1.89)\end{array}$ & $\begin{array}{c}3.0 \\
(1.84)\end{array}$ & $\begin{array}{c}2.7 \\
(1.77)\end{array}$ & $\begin{array}{c}2.7 \\
(1.77)\end{array}$ & $\begin{array}{c}2.4 \\
(1.71)\end{array}$ & $\begin{array}{c}2.3 \\
(1.67)\end{array}$ & $\begin{array}{c}2.2 \\
(1.64)\end{array}$ & $\begin{array}{c}2.0 \\
(1.58)\end{array}$ & $\begin{array}{c}1.8 \\
(1.51)\end{array}$ \\
\hline $\mathbf{T}_{7}$ & Chlorpyriphos 20EC @2ml/lit. & $\begin{array}{c}5.1 \\
(2.37)\end{array}$ & $\begin{array}{c}4.8 \\
(2.29)\end{array}$ & $\begin{array}{c}3.7 \\
(2.03)\end{array}$ & $\begin{array}{c}2.3 \\
(1.68)\end{array}$ & $\begin{array}{c}0.3 \\
(0.91)\end{array}$ & $\begin{array}{c}1.2 \\
(1.31)\end{array}$ & $\begin{array}{c}3.1 \\
(1.90)\end{array}$ & $\begin{array}{c}3.0 \\
(1.87)\end{array}$ & $\begin{array}{c}2.3 \\
(1.68)\end{array}$ & $\begin{array}{c}1.3 \\
(1.34)\end{array}$ & $\begin{array}{c}0.3 \\
(0.91)\end{array}$ & $\begin{array}{c}1.3 \\
(1.35)\end{array}$ & $\begin{array}{c}2.3 \\
(1.68)\end{array}$ \\
\hline $\mathbf{T}_{8}$ & Untreated control. & $\begin{array}{c}5.3 \\
(2.38)\end{array}$ & $\begin{array}{c}5.3 \\
(2.36)\end{array}$ & $\begin{array}{c}5.3 \\
(2.40)\end{array}$ & $\begin{array}{c}5.0 \\
(2.32)\end{array}$ & $\begin{array}{c}5.0 \\
(2.30)\end{array}$ & $\begin{array}{c}4.6 \\
(2.24)\end{array}$ & $\begin{array}{c}4.8 \\
(2.28)\end{array}$ & $\begin{array}{c}4.8 \\
(2.26)\end{array}$ & $\begin{array}{c}4.4 \\
(2.21)\end{array}$ & $\begin{array}{c}4.0 \\
(2.10)\end{array}$ & $\begin{array}{c}3.3 \\
(1.95)\end{array}$ & $\begin{array}{c}3.0 \\
(1.86)\end{array}$ & $\begin{array}{c}3.1 \\
(1.83)\end{array}$ \\
\hline & $\mathrm{SE}(\mathrm{m}) \pm$ & 0.14 & 0.14 & 0.15 & 0.14 & 0.13 & 0.14 & 0.13 & 0.12 & 0.13 & 0.12 & 0.11 & 0.09 & 0.14 \\
\hline & C.D. at $5 \%$ & N S & 0.42 & 0.45 & 0.42 & 0.39 & 0.42 & 0.39 & 0.36 & 0.39 & 0.36 & 0.33 & 0.27 & 0.42 \\
\hline & C.V. & 10.24 & 11.49 & 12.63 & 12.92 & 14.78 & 14.75 & 11.37 & 11.51 & 13.17 & 13.16 & 14.55 & 10.42 & 13.79 \\
\hline
\end{tabular}

Fig. in parenthesis are $\sqrt{x}+0.5$ transformed values. 
Table.2 Management of S.litura on soybean through bio-rational approaches

\begin{tabular}{|c|c|c|c|c|c|c|c|c|c|c|c|c|c|c|}
\hline \multirow{3}{*}{$\begin{array}{l}\text { Tr } \\
\text { No }\end{array}$} & \multirow[t]{3}{*}{ Treatments } & \multicolumn{13}{|c|}{ Average larvae/mrl } \\
\hline & & \multicolumn{6}{|c|}{ Third spray } & \multicolumn{7}{|c|}{ Simple pooled mean of three sprayings } \\
\hline & & 1DAS & 3DAS & 5DAS & 7DAS & 10DAS & 14DAS & 1DBS & 1DAS & 3DAS & 5DAS & 7DAS & 10DAS & 14DAS \\
\hline $\mathbf{T}_{1}$ & NSKE $5 \%$ & $\begin{array}{c}2.7 \\
(1.76)\end{array}$ & $\begin{array}{c}2.3 \\
(1.68)\end{array}$ & $\begin{array}{c}1.9 \\
(1.54)\end{array}$ & $\begin{array}{c}0.7 \\
(1.07)\end{array}$ & $\begin{array}{c}0.6 \\
(1.02)\end{array}$ & $\begin{array}{c}0.6 \\
(1.02)\end{array}$ & $\begin{array}{c}5.0 \\
(2.34)\end{array}$ & $\begin{array}{c}3.5 \\
(2.00)\end{array}$ & $\begin{array}{c}3.1 \\
(1.88)\end{array}$ & $\begin{array}{c}2.2 \\
(1.64)\end{array}$ & $\begin{array}{c}0.7 \\
(1.11)\end{array}$ & $\begin{array}{c}1.2 \\
(1.29)\end{array}$ & $\begin{array}{c}2.2 \\
(1.60)\end{array}$ \\
\hline $\mathbf{T}_{2}$ & Neem oil $5 \%$ & $\begin{array}{c}2.8 \\
(1.79)\end{array}$ & $\begin{array}{c}2.3 \\
(1.68)\end{array}$ & $\begin{array}{c}2.0 \\
(1.58)\end{array}$ & $\begin{array}{c}1.1 \\
(1.27)\end{array}$ & $\begin{array}{c}0.8 \\
(1.13)\end{array}$ & $\begin{array}{c}0.6 \\
(1.02)\end{array}$ & $\begin{array}{c}5.2 \\
(2.39)\end{array}$ & $\begin{array}{c}3.7 \\
(2.04)\end{array}$ & $\begin{array}{c}3.1 \\
(1.89)\end{array}$ & $\begin{array}{c}2.3 \\
(1.67)\end{array}$ & $\begin{array}{c}0.9 \\
(1.20)\end{array}$ & $\begin{array}{c}1.3 \\
(1.35)\end{array}$ & $\begin{array}{c}2.4 \\
(1.65)\end{array}$ \\
\hline $\mathbf{T}_{3}$ & Pongamia oil $2 \%$ & $\begin{array}{c}3.1 \\
(1.88)\end{array}$ & $\begin{array}{c}2.8 \\
(1.80)\end{array}$ & $\begin{array}{c}2.3 \\
(1.66)\end{array}$ & $\begin{array}{c}1.3 \\
(1.35)\end{array}$ & $\begin{array}{c}0.8 \\
(1.13)\end{array}$ & $\begin{array}{c}0.7 \\
(1.08)\end{array}$ & $\begin{array}{c}5.3 \\
(2.41)\end{array}$ & $\begin{array}{c}3.9 \\
(2.10)\end{array}$ & $\begin{array}{c}3.3 \\
(1.95)\end{array}$ & $\begin{array}{c}2.6 \\
(1.76)\end{array}$ & $\begin{array}{c}1.1 \\
(1.25)\end{array}$ & $\begin{array}{c}1.5 \\
(1.41)\end{array}$ & $\begin{array}{c}2.6 \\
(1.71)\end{array}$ \\
\hline $\mathbf{T}_{4}$ & Mechanical control. & $\begin{array}{c}0.3 \\
(0.91)\end{array}$ & $\begin{array}{c}0.6 \\
(1.02)\end{array}$ & $\begin{array}{c}1.0 \\
(1.22)\end{array}$ & $\begin{array}{c}1.4 \\
(1.39)\end{array}$ & $\begin{array}{c}0.9 \\
(1.17)\end{array}$ & $\begin{array}{c}0.8 \\
(1.13)\end{array}$ & $\begin{array}{c}5.3 \\
(2.17)\end{array}$ & $\begin{array}{c}0.4 \\
(0.93)\end{array}$ & $\begin{array}{c}0.6 \\
(1.05)\end{array}$ & $\begin{array}{c}0.9 \\
(1.20)\end{array}$ & $\begin{array}{c}1.4 \\
(1.38)\end{array}$ & $\begin{array}{c}1.8 \\
(1.49)\end{array}$ & $\begin{array}{c}2.8 \\
(1.75)\end{array}$ \\
\hline $\mathbf{T}_{5}$ & Use of trap crop, castor. & $\begin{array}{c}1.1 \\
(1.27)\end{array}$ & $\begin{array}{c}0.9 \\
(1.16)\end{array}$ & $\begin{array}{c}1.0 \\
(1.22)\end{array}$ & $\begin{array}{c}0.9 \\
(1.18)\end{array}$ & $\begin{array}{c}0.8 \\
(1.12)\end{array}$ & $\begin{array}{c}0.3 \\
(0.91)\end{array}$ & $\begin{array}{c}4.2 \\
(2.17)\end{array}$ & $\begin{array}{c}2.4 \\
(1.66)\end{array}$ & $\begin{array}{c}2.2 \\
(1.60)\end{array}$ & $\begin{array}{c}2.1 \\
(1.57)\end{array}$ & $\begin{array}{c}1.8 \\
(1.48)\end{array}$ & $\begin{array}{c}1.6 \\
(1.42)\end{array}$ & $\begin{array}{c}1.2 \\
(1.26)\end{array}$ \\
\hline $\mathbf{T}_{6}$ & $\begin{array}{l}\text { Intercrop ( } 4 \text { rows of } \\
\text { soybean } x 2 \text { rows of } \\
\text { sorghum). }\end{array}$ & $\begin{array}{c}1.7 \\
(1.46)\end{array}$ & $\begin{array}{c}1.6 \\
(1.43)\end{array}$ & $\begin{array}{c}1.6 \\
(1.43)\end{array}$ & $\begin{array}{c}1.4 \\
(1.39)\end{array}$ & $\begin{array}{c}1.2 \\
(1.31)\end{array}$ & $\begin{array}{c}0.7 \\
(1.08)\end{array}$ & $\begin{array}{c}4.2 \\
(2.17)\end{array}$ & $\begin{array}{c}2.9 \\
(1.81)\end{array}$ & $\begin{array}{c}2.7 \\
(1.77)\end{array}$ & $\begin{array}{c}2.6 \\
(1.73)\end{array}$ & $\begin{array}{c}2.2 \\
(1.64)\end{array}$ & $\begin{array}{c}2.1 \\
(1.59)\end{array}$ & $\begin{array}{c}1.7 \\
(1.47)\end{array}$ \\
\hline $\mathbf{T}_{7}$ & $\begin{array}{l}\text { Chlorpyriphos 20EC } \\
\text { @ 2ml/lit. }\end{array}$ & $\begin{array}{c}2.1 \\
(1.61)\end{array}$ & $\begin{array}{c}1.6 \\
(1.43)\end{array}$ & $\begin{array}{c}1.0 \\
(1.22)\end{array}$ & $\begin{array}{c}0.3 \\
(0.91)\end{array}$ & $\begin{array}{c}0.6 \\
(1.02)\end{array}$ & $\begin{array}{c}0.7 \\
(1.09)\end{array}$ & $\begin{array}{c}5.1 \\
(2.37)\end{array}$ & $\begin{array}{c}3.3 \\
(1.93)\end{array}$ & $\begin{array}{c}2.5 \\
(1.72)\end{array}$ & $\begin{array}{c}1.5 \\
(1.41)\end{array}$ & $\begin{array}{c}0.3 \\
(0.89)\end{array}$ & $\begin{array}{c}1.0 \\
(1.23)\end{array}$ & $\begin{array}{c}2.0 \\
(1.56)\end{array}$ \\
\hline $\mathbf{T}_{8}$ & Untreated control. & $\begin{array}{c}3.0 \\
(1.86)\end{array}$ & $\begin{array}{c}3.0 \\
(1.85)\end{array}$ & $\begin{array}{c}2.6 \\
(1.73)\end{array}$ & $\begin{array}{c}2.3 \\
(1.66)\end{array}$ & $\begin{array}{c}1.6 \\
(1.42)\end{array}$ & $\begin{array}{c}1.3 \\
(1.34)\end{array}$ & $\begin{array}{c}5.3 \\
(2.38)\end{array}$ & $\begin{array}{c}4.4 \\
(2.19)\end{array}$ & $\begin{array}{c}4.2 \\
(2.16)\end{array}$ & $\begin{array}{c}3.9 \\
(2.08)\end{array}$ & $\begin{array}{c}3.5 \\
(1.99)\end{array}$ & $\begin{array}{c}3.1 \\
(1.86)\end{array}$ & $\begin{array}{c}3.1 \\
(1.85)\end{array}$ \\
\hline & $\mathrm{SE}(\mathrm{m}) \pm$ & 0.13 & 0.12 & 0.10 & 0.10 & 0.08 & 0.05 & 0.14 & 0.07 & 0.08 & 0.08 & 0.10 & 0.06 & 0.06 \\
\hline & C.D. at $5 \%$ & 0.39 & 0.36 & 0.30 & 0.30 & 0.24 & 0.15 & $\mathbf{N} \mathbf{S}$ & 0.22 & 0.25 & 0.24 & 0.30 & 0.19 & 0.18 \\
\hline & C.V. & 14.19 & 13.84 & 11.81 & 13.38 & 11.61 & 8.01 & 10.24 & 7.07 & 8.37 & 8.59 & 12.72 & 7.80 & 6.44 \\
\hline
\end{tabular}

Fig. in parenthesis are $\sqrt{ } \mathrm{x}+0.5$ transformed values. DBS-Day before spraying DAS-Day after spraying 
Int.J.Curr.Microbiol.App.Sci (2018) 7(12): 216-222

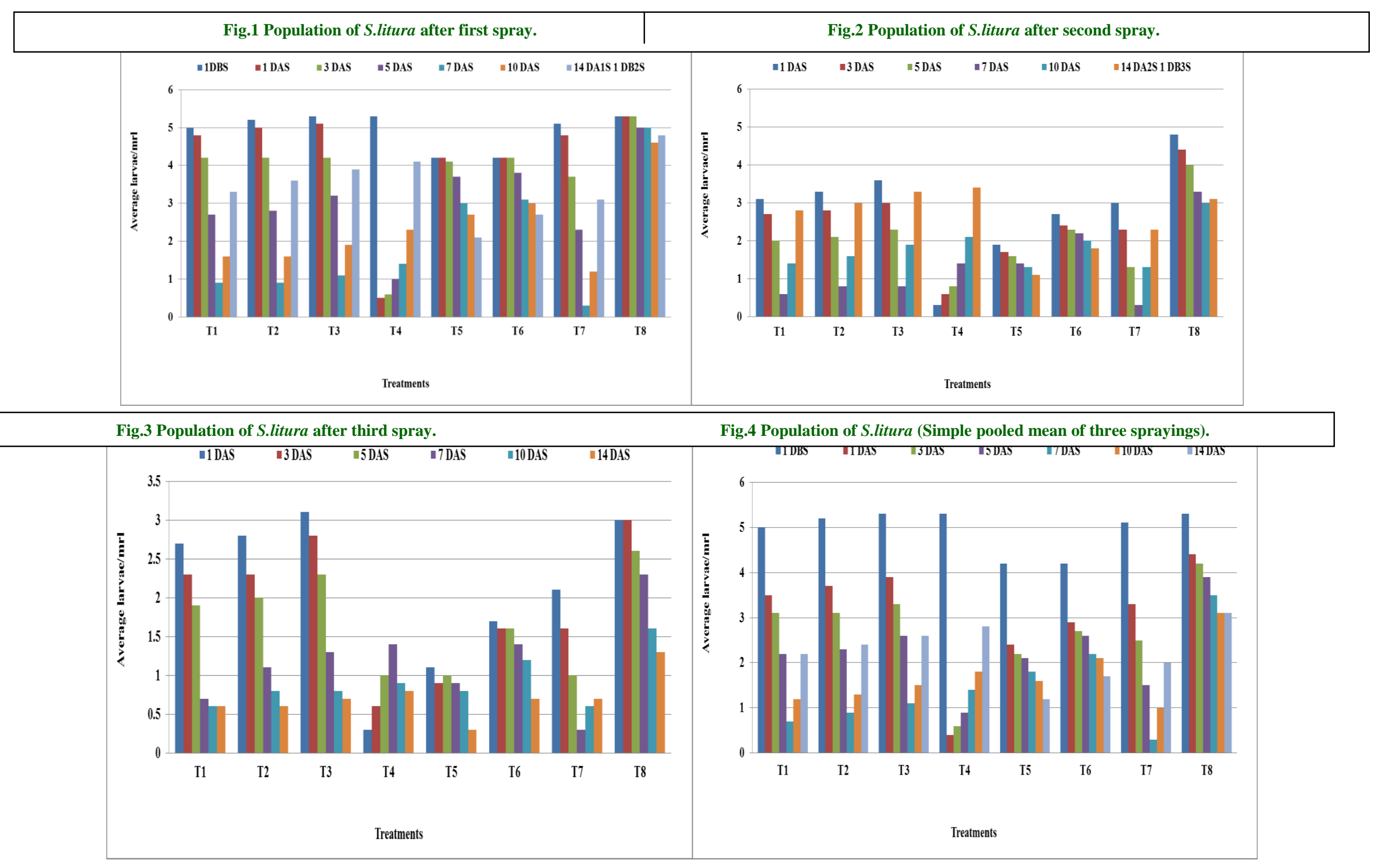


After second spray the larval population significantly varied among the treatments, highest larval population was recorded in untreated control where chlorpyriphos 20EC @2 $\mathrm{ml} / \mathrm{lit}$ recorded the lowest larval population and at par with NSKE 5\% and Neem oil $5 \%$. But pongamia oil $2 \%$ treated plot recorded highest larval population. At seventh day after spraying similar trend was noticed. The lowest S.litura were recorded in mechanical control $1^{\text {st }}, 3^{\text {rd }}, 5^{\text {th }}$ and $7^{\text {th }}$ day after II $^{\text {nd }}$ mechanical controls. Trap crop (castor) were found most effective in reducing S.litura larvae and lowest S.litura (1.1 larvae/mrl) were recorded in fourteenth days after II $^{\text {nd }}$ spray as shown in (Table 1 and Fig. 2).

After third spray chlorpyriphos 20EC @2 $\mathrm{ml} /$ lit was significantly superior over all other treatments after first, third, fifth, seventh, tenth days after spraying and at par with NSKE 5\% and Neem oil 5\%. The lowest Spodoptera were recorded in mechanical control $1^{\text {st }}, 3^{\text {rd }}, 5^{\text {th, }}$ and $7^{\text {th }}$ day after III $^{\text {nd }}$ mechanical controls. Trap crop (castor) were found most effective in lowest S.litura (0.3 larvae/mrl) were recorded in fourteenth days after III $^{\text {nd }}$ spray as shown in (Table 2 and Fig. $3)$.

The pooled data of three sprayings presented in (Table 2 and Fig. 4) revealed that. The larval population of S.litura was uniformly distributed in all the plots one day before spray. However significant reduction in the larval population was recorded $\mathrm{I}^{\text {st }}, 3^{\text {rd }}$ and $5^{\text {th }}$ days after treatment of III $^{\text {rd }}$ mechanical control while lowest larval population of S.litura registered with the treatment of chlorpyriphos 20EC @2ml/lit after $7^{\text {th }}$ and $10^{\text {th }}$ day after spray and which was at par with the NSKE 5\%. However the least larval activity of S.litura noticed in the treatment of trap crop (castor) followed by chlorpyriphos 20EC@2ml/lit and NAKE 5\%.
Sharma (2005) reported that, the hand picking reduced the S.litura population in soybean. Bhosle et al., (2008) reported that the localized IPM module for soybean pests which collection and destruction of tobacco leaf eating caterpillar along with leaves effective in managing the pest. Nath et al., (2011) studied that the azadirachtin was found to reduce feeding behavior of S.litura on soybean. Hence the present findings are in agreement with the earlier work of above scientist.

\section{References}

Adak, T., Kumar, J., Dey, D., Shakil, N.A and Walia, S. (2012) Residue and bioefficacy evaluation of controlled release formulation of imidacloprid against pests in soybean (Glycine max). $J$. Environmental Sci. and Health, 47: 226-231.

Andole, V.C. (1984) "Soybean, It's cultivation, uses and values in dietetics.” pp 29.

Ansari, M.M. and A.N. Sharma, (2005) Compatibility of Bacillus thuringiensis and Beauveria bassiana with new insecticides recommended for insect pest control in soybean. Pestology, 29(9): 18-20.

Bhosle, B.B., Bhede, B.V., Patange, N.R., Kambale, S.K. and Baig, K.S., (2008) IPM in soybean, M.A.U. Extn. Folder No. 2/2008.

Biswas, K., Chattopadhyay, I., Banerjee, R.K., Bandyopadhyay, U. (2002) Biological activities and medicinal properties of neem (Azadirachta indica). Curr. Sci 82: 1336-1345.

Luckmann, W.H. (1971) The insect pests of soybean. World farm, 13(5): 18-19 \& 22.

Munde, D.R. (1980) Insect pest complex on soybean Glycine max (L.) in Marathwada region. J. Maharashtra 
Agric. Univ., 5(3): 259-261.Kumawal, M.M and Kumar, A. 2007. Bioefficacy of some novel insecticides against Jassids in Soybean. Pestology. 31(2): 29-32.
Nath, G. and Singh, K. (2011) Effect of Foliar Sprays of Biopesticides and Vermiwash of Animal, Agro and Kitchen Wastes on Soybean (Glycine max L.) crop.

NNagata, T. (1960) Sci. Report. Ser.Agri. Hyogo. Univ. Agril., 4: 101-104.

\section{How to cite this article:}

Misal, M.R., B.V. Patil and Khaire, P.B. 2018. Management of Spodoptera litura in Soybean through Biorational Approaches. Int.J.Curr.Microbiol.App.Sci. 7(12): 216-222.

doi: https://doi.org/10.20546/ijcmas.2018.712.027 